\title{
Palliative interventions in outpatient dialysis network
}

\author{
Ana Branco ${ }^{1}$, Ana Farinha ${ }^{2}$, Ana Mateus ${ }^{3}$, Cristina Outerelo ${ }^{3}$, Pedro Ponce ${ }^{4}$ \\ ${ }^{1}$ NephroCare Santa Maria da Feira \\ ${ }^{2}$ NephroCare Setúbal \\ ${ }^{3}$ NephroCare Santarém \\ ${ }^{4}$ Country Medical Director, NephroCare Portugal \\ Names are presented in alphabetic order, since all authors had similar contributions
}

\section{ABSTRACT}

Chronic kidney disease (CKD) is a burdensome disease that has been successfully managed with dialysis. But even if dialysis can prolong patients' survival in certain circumstances, it also brings a high burden of physical and psychosocial symptoms, poor outcomes, and high costs of care. Fragile patients may not benefit from starting dialysis. In this way, Palliative Care (PC) should be integrated into CKD care to control symptoms' burden, to discuss the shared decision to start or forgo dialysis when stage 5 CKD approaches, or to select conservative management of end-stage renal disease (ESRD) or withdrawal dialysis. As patients attended in private hemodialysis units get older and more fragile, incorporation of PC is associated with the highest quality care standards provided to our ESRD patients. That being so, we designed a project based on the World Health Organization (WHO) recommendations to implement palliative interventions in NephroCare Hemodialysis Units. In our project, we consider the following five steps: (i) assessment of palliative needs in dialysis patients; (ii) team education; (iii) contact and referral to specialized teams; (iv) registration ( $v$ ) and trial of the pilot project. We also reflect on the barriers to implementation of this project. This article aims to raise awareness of the project and use it as a model or a first step to other projects in the actual area to be implemented in other health units.

Keywords: Chronic kidney disease, Palliative Care, Project Implementation

\section{INTRODUCTION}

As a result of many years of accumulated knowledge and experience, it is now realized that dialysis (a life-saving and life-prolonging treatment), when applied to end stage renal disease (ESRD) replaces only a modest portion of all the physiological wonders of renal function and does not relieve or cure the always present underlying organ failures.

Dialysis is nowadays a routine medical procedure offered to a population growing older, which in most cases displays an increasing number of comorbidities, decreased life expectancy and often has cognitive decline over time, as the disease progresses ${ }^{1,2}$. There is growing evidence that many patients over 75 may not benefit from dialysis therapy. In the United States (US), as well as in Portugal, maintaining dialysis for those patients has doubled over the last two decades ${ }^{3}$.

In many countries, up to $15 \%$ of all incident ESRD patients choose conservative treatment (that is, without any form of dialysis and only palliative care) as their treatment modality ${ }^{4,5}$. Many studies evidence that conservative treatment should be discussed not as a last resort but as an option that might be most effective in promoting patient goals $^{6}$.

In Portugal, although the health authorities have approved and given clear support to this conservative modality (guidelines from
Direção Geral de Saúde, 2011) ${ }^{7}$, to our knowledge that resolution did not translate into either an increased number of patients selecting the conservative option or to a structured preparation of the nephrology services in public hospitals to respond to the specific needs of these patients and their families. For those reasons, in private hemodialysis units such as NephroCare Portugal, we continue to receive incident patient referrals to our outpatient dialysis units network; patients in whom for which hemodialysis is certainly against their best interest and wellbeing.

Conversely in many countries, treatment withdrawal in prevalent patients undergoing hemodialysis is the second or third most frequent cause of death. Withdrawing dialysis is more likely in countries in which palliative care is available and reimbursed, and is provided in public institutions. The number of patients choosing withdrawal increases with the level of training and education of local nephrologists ${ }^{8}$. In Portugal, the conservative approach, although in line with our present by-laws, is rarely chosen, in part due to a precarious infrastructure to support patients and their families. The answer requires that physicians and nephrologists offer their patients alternative solutions, namely a well-designed palliative intervention.

For the United States Centers for Medicare and Medicaid Services (US.CMS), a well-designed Paliative Care (PC) is a patient and family-centered care that optimizes the patient quality of life by anticipating, preventing, and treating suffering ${ }^{9}$. As stated by Tamura and coworkers, 
a palliative approach to dialysis care is a transition from a conventional disease-oriented focus on dialysis as a rehabilitative treatment to an approach prioritizing comfort and alignment with patient preferences and goals of care, to improve quality of life and reduce symptom burden in patients' final year of life $e^{10}$.

Concerned with the situation in Portugal, as described above, NephroCare Portugal (with 38 dialysis units in Portugal), implemented a specific project (already being tested in four dialysis units), based on an integrated approach for palliative intervention to support all patients and their families who choose to withhold or withdraw from dialysis treatment at some stage of their disease.

\section{PROJECT DESCRIPTION}

The Palliative Intervention project in NephroCare outpatient hemodialysis units includes:

1. Assessment of palliative needs in dialysis patients

2. Team education and training

3. Interdisciplinary work with specialized teams

4. A dedicated electronic health record for palliative intervention

5. Outcomes measurement and auditing

The authors describe the project stages below.

\section{Assessment of palliative needs in dialysis patients}

According to the United Nations Committee on Economic, Social, and Cultural Rights, it is essential to provide "attention and care to chronically and terminally ill persons, sparing them avoidable pain, and enabling them to die with dignity"11. Moreover, the World Health Organization states that palliative care promotes quality of life of patients and their families through the prevention and relief of suffering, using early identification and faultless assessment and treatment of pain and other physical, psychosocial, and spiritual ailments.

ESRD in this population is not a single disease and patients must manage the demands of dialysis therapy as well as those associated with other comorbid conditions and symptoms.

The first palliative need is the proper assessment of symptoms.

Illness burden and quality of life can only be evaluated through patient self-reporting. Unfortunately, many patients lose their ability to cooperate in such a crucial step of the decision-making processes. Also, the disease trajectories of chronic kidney failure may be quite different from patient to patient, making the integration of palliative discussions a difficult task. So, the second need we identified was early initiation of the shared decision-making processes and the discussion of end of life preferences ${ }^{12}$.

\section{Assessment of symptom burden in dialysis patients}

Patients with ESRD have a high symptom burden that most commonly includes high rates of fatigue, dyspnea, insomnia, pain, pruritus, anxiety and depression, notably affecting their health-related quality of life $\mathrm{e}^{13,14}$. In the dialysis population, these symptoms are often underrecognized and undertreated. Palliative care in this setting is therefore expected to decrease patient suffering. It can be assessed using validated tools such as the 17-item Palliative Outcome Scale Symptom "IPOS Renal"15. We implemented the use of that scale as routine in our dialysis patients' management, which may allow clinicians to adjust medication and interventions to patients' needs. Interestingly, a dramatic change over time is noted in the severity and presence of symptoms, highlighting the need for regular surveillance in our dialysis patients ${ }^{16}$.

\section{Shared decision-making processes and discussing end of life preferences}

The decision to start dialysis or maintain conservative management is often complex and involves patient priorities and goals, clinical considerations such as the rate of decline and renal failure symptoms, as well as the projected prognosis for each option ${ }^{17}$. Following the above reasoning, the prognosis should always be discussed with the patients. To access prognosis, we implemented validated tools to be used by the dialysis population: However, there are as yet no validated tools for estimating prognosis in conservative management for CKD. Although this makes it challenging to compare prognosis in both scenarios, a few scores have been used in individual patients. The Rein score is recommended to predict survival at six months in elderly incident patients starting dialysis ${ }^{18}$ and the Cohen score to predict patient mortality at six months in prevalent patients already in hemodialysis ${ }^{19}$.

In addition to prognosis, another point that should be discussed with patients is quality of life, which seems to be highly affected after starting dialysis, with lower levels reported, but that remains stable in conservative treatment, although also in low levels ${ }^{20}$.

Thus, palliative care should be offered to all patients that suffer from the hardships of their disease, whatever the stage or treatment option. To access the patient's perception of their own quality of life (which can be quite different from clinical staff opinion), we used the HRQOL EQ-5D-5L score as part of our routine delivery of patient-centered care $^{21}$.

In addition to the patient's perspective, dialysis facilities should also identify patients that are no longer benefiting from dialysis therapy because their clinical condition is progressively declining. To target these patients, generating a "cause of concern register", a cornerstone of this project, we selected major trigger events that run up a red flag:

- More than two hospital inpatient admissions in 3 months;

- Weight loss $>10 \%$ in 6 months, or albumin $<2.5 \mathrm{~g} / \mathrm{d}$;

- Dialysis intolerance or recurrent vascular access problems;

- Institutionalization or progressive decline in functional status (Karnofsky index $\leq 40 \%$; Frailty score $\geq 6$ );

- The answer to the Surprise Question "would you be surprised if this patient died in the next 6 months?"

These events should prompt conversations about those patients' goals of care 22 . Withdrawing from dialysis may be considered in patients with a poor functional status that is deteriorating despite 
optimized dialysis treatment, either because of malignancy or a neuro-degenerative condition, along with a High Comorbidity index and a NO answer to the Surprise Question ${ }^{23}$. This decision is not a moment but a process, which may involve reducing the time and frequency of sessions, palliative dialysis, or total suspension. For patients requiring dialysis but whose prognosis is uncertain, or for whom a consensus has not been reached, a self-limited trial of dialysis with a well-defined timeframe and clear clinical targets is also advocated. It is of utmost importance that dialysis facilities be aware of the need to reevaluate these patients regularly.

To guide nephrologists through these difficult options, in 2010, the Renal Physicians Association (RPA) and the American Society of Nephrology updated the guidelines on Shared Decision-Making in the Appropriate Initiation of and Withdrawal from Dialysis. This document clarifies that it is appropriate to forgo dialysis for patients that refuse dialysis as well as for patients who have a very poor prognosis or to whom dialysis cannot be provided safely ${ }^{24}$. The RPA suggested as criteria to propose withholding, or withdrawing dialysis the following patient phenotypes: Severe and irreversible dementia; Permanently unconscious; Confined to bed or chair; Dependent for basic activities of daily living; Unable to cooperate with treatment; End-stage lung, liver or heart disease; Non-feasibility of dialysis treatment (unavailable vascular access, refractory hypotension).

Patients' goals and values should be respected and the decision to forgo or withdraw dialysis after the trial should be reassessed by clinicians, requiring well-trained communication skills in difficult conversations with patients, or more commonly, their family.

\section{Team Education and training (Palliative Care Com- petencies in Nephrologyl}

One of the pillars of the public health strategy for palliative care is education ${ }^{25}$. This covers the education of professional, nonprofessional team members, and laypersons (semi-skilled home-based caregivers or family caregivers).

All elements that constitute the core of the project team have formal and previous training in Palliative Care. Our next goal is to broadly disseminate education to other members of our staff 26 .

The European Association of Palliative Care recommends that practitioners should be prepared academically according to three levels of specialization ${ }^{27}$. At the first level - Palliative Care Approach - all healthcare professionals should receive education on the principles and practices of palliative care. The second and third levels - General Palliative Care and Specialist Palliative Care - should provide the specialist level of knowledge.

General palliative care is an intermediate level expertise targeting physicians, who although not engaged regularly in palliative care, had some additional training and experience in palliative care. This is a competence much needed in disciplines like nephrology and cardiology, where professionals face complex and frequent end of life situations.
Finally, Specialist Palliative Care is intended for professionals working solely in the field of palliative care, that involve the care of patients with more complex and demanding care needs, thus requiring a higher degree of training, a multidisciplinary team, and other resources.

It is imperative that we agree on the core competencies (Level 1 and 2) required for nephrologists and co-workers to treat patients with advanced kidney disease. The required skills and training structure were accredited both by the national societies of palliative care and nephrology to empower and give credibility to the trainees.

Competencies provided at the generalist level were: (i) Communication and interpersonal skills, as cornerstones of the physician-patient relationship. For example, nephrologists should be able to promote patient-family meetings and to clearly and effectively communicate a grim prognosis, or complex plans of care. (ii) Assess and manage basic and prevalent symptoms, not just the medical treatment, but also master the holistic approach that include "psychosocial and spiritual support"; (iii) Ability to have basic appropriate advanced care conversations with patients and family, reaching a consensus on the goals of treatment, suffering relief, discussing resuscitation and intensive care admission in the event of further deterioration; (iv) Capability to deal with ethical issues in dialysis decision-making; (v) Providing end of life care; (vi) To practice comprehensive care co-ordination and interdisciplinary teamwork ${ }^{28}$.

Also of vital importance will be to inform and share our program and goals with the health authorities, as well as our professional leaders, let them know about the benefits of a proficient palliative intervention, affording dignity, comfort and respect at the end of life 29,30 . The benefits extend to all stakeholders, with a considerable money-saving opportunity to the payer and opening a new care modality to the provider that better serves its patients.

Currently, formal palliative care education in Portugal remains sparse. Palliative medicine as a postgraduate specialty course is not yet accredited. The national societies of palliative care and nephrology must persuade the Portuguese Medical Association to license nephrology palliative intervention as a renal subspecialty, to be included in the core curriculum of undergraduate and post-graduate nephrology training.

\section{Interdisciplinary work with specialized teams}

Outpatient hemodialysis units are already examples of multidisciplinary teams, including among their staff doctors, nurses, social workers, psychologists, pharmacists and nutritionists. As stated previously, team members have basic training in palliative care, and all these resources have been working together to identify patients who might benefit the most from palliative interventions. Complex cases will still be handled by Palliative Care specialists. Therefore, we approached the Comissão Nacional de Cuidados Paliativos to present our project and prepare a palliative care referral network both in public hospitals and in the community. It is our intention to create a fast-track referral to those patients requiring complex interventions, including the need for hospitalization in hospice care (Unidades de Cuidados Paliativos). 


\section{A dedicated electronic health record for palliative intervention}

The project was implemented in four pilot centers. Patients were assessed according to the palliative needs previously identified.

Information leaflets were distributed to patients/families to introduce this intervention, possible benefits, and introduce discussion topics.

All this activity is registered in a dedicated electronic health records (EHR), satellite to our general database, European Clinical Database - EuCliDC (Fresenius Medical Care - Bad Homburg).

This IT tool allows us to identify patients who might benefit from palliative intervention.

Our main focus lies in: (i) regular follow-up, also performed in our EHR, (ii) built-in assessment scales for most domains of interest in a palliative clinic (HRQOL, (iii) mortality prediction, (iv) symptom inventory, (v) comorbidity index, (vi) frailty and functional scores, (vii) and being alert to changes in patients' clinical situations.

Registries are useful as well to monitor our interventions and their results, or to validate tools that have not been used so far in the Portuguese ESRD population.

Clinical assessments have been separated into folders for incident patients (patients who are on dialysis for less than 90 days) and for prevalent patients.

Incident patients (which we hadn't treated prior to their attendance at our unit) are assessed based on the Modified Charlson Comorbidity Index and on "the surprise question". Prevalent patients at risk are identified using sentinel events, and at least one of the following should be present: (i) more than two hospital inpatient stays in 3 months; (ii) weight lost $>10 \%$ in six months or albumin $<2.5 \mathrm{~g} / \mathrm{dL}$; (iii) dialysis intolerance or recurrent vascular problems; (iv) institutionalization or decrease in functional status; ( $v$ ) the surprise question which predict alterations in their functional and clinical status.
After identifying a patient at risk, possible evaluation by the local Fresenius Palliative Team is proposed. If the patient (or family) agrees to attend this clinic, written information is provided, and a formal evaluation is done.

Patients are assessed for their needs, prognosis, and care planning, based on well-validated palliative recommendations.

All these assessments and shared decisions are recorded in the same dedicated program and may be consulted by all members of the team.

\section{Outcomes measurements and auditing}

The last phase of the project is to audit the program, which presents us with a chance for improvement. Since our goal is to develop End of Life (EoL) care for our patients, it is crucial to evaluate the impact of this project on patients' quality of life and quality of dying ${ }^{31}$. With the premise "if you don 't measure it, you won't improve it" in mind, we propose to Plan-Do-Check and Act (PDCA) ${ }^{32}$. The main goals to audit are to check if the patients were correctly identified and whether their and their caregivers' needs have been assessed and met. Analysing all causes of death in our dialysis patients will help us to understand if the tools described above are useful in a Portuguese population with ESRD in identifying those in the last year of life.

We have learned from previous palliative care studies that from the patient's perspective some of their most important priorities are to receive good pain and symptoms' control; avoid unnecessary prolongation of death and to relief the burden on loved ones ${ }^{33}$. From the family's perspective, it is important to feel supported in practical issues and in bereavement; be involved in decisions, without feeling guilty, as many of our patients do not retain any longer a sound decision capacity; to avoid patients' suffering and to respect their wishes. Nephrologists must be aware of patients' wishes as well as their preferred place to die. Dialysis units should be responsible for putting the EoL care pathway in place, to know and connect with local resources and to link the different actors involved.

Qualitative interviews to caregivers one month after death, by phone or in person, based on already existing toolkits ${ }^{34}$, should be undertaken.

\section{Table 1}

Domains to audit

Patient

Dialysis Unit
Was there a good control of symptoms?

Was there a care plan in place (medication adjusted; change in dialysis prescription; Food and hydration addressed)?

Was the dying time prolonged unnecessary? Did the patient die in his preferred place of death?

Were you aware of the prognosis and involved in shared decisions?

Did you feel supported (emotional and practical aspects)?

Did you feel your relative died in peace? Do you think it was the appropriate place to die?

Looking at all death in $1 / y$, was the patient recognized as a cause of concern?

Was correspondence with the surprise question and prognosis scores accurate?

Were advanced directives, such as "no resuscitation", registered in patient's report?

Was the local palliative care team involved? Were the local resources adequate?

What was the time of response from the "red flag on" and time to death? 
Quantitative and quality parameters, as expressed in Table 1, must be checked once a year, by the audit group (named by each clinic), discussed internally, and shared with other units.

\section{BARRIERS TO IMPLEMENTATION}

The most relevant barriers to a successful implementation of a conservative treatment and palliative intervention program in the outpatient dialysis setting is the lack of renal physicians training as well as economic and regulatory factors. Our National Health Service, the universal payer for the whole ESRD treatment program, establishes targets for dialysis care through performance standards and providers frequently include incentives to ensure an optimal level of care. Incentivized metrics are quite often in conflict with individualized patient-centered care, generating financial penalties for unmet metrics. The lack of a wide-scale infrastructure, or a fair and well-regulated reimbursement policy for providing palliative support services to dialysis patients, also contributes to our current situation ${ }^{2}$

Other hurdles in our way include: i) Physicians are reluctant to deprive their patients and families of their last hope for recovery; ii) It is easier, faster, and more profitable to provide full dialysis treatment than to have a difficult and long-lasting conversation about prognosis and end of life planning; iii) We always doubt what our patients really understand from our explanations, their motivation to forgo dialysis, and how stable their preferences and decisions will be; iv) There is a lack of training and experience in difficult conversations with our patients and the timing never seems to be the right one; $v$ ) We want to avoid family litigation, conflicts with the referral nephrologists, to frustrate our management expectations, or our own interests; vi) It is of utmost relevance to face specific costs for this modalities, such as home visits and care for those unable to travel to the dialysis unit; purchasing and distribution of medication; patient transportation; and palliative care specialist consult.

\section{CONCLUSIONS}

There is no doubt that, in general, dialysis is associated with a significant survival advantage, but this advantage is dramatically reduced for older people (mostly above 75 years) with major comorbidity (mainly coronary artery disease), or poor functional status, and may be lost if we only account for hospital free survival and quality of life ${ }^{4}$.

The majority of older patients are too overwhelmed by their chronic conditions and frailties to engage in the big questions raised by the beginning of dialysis. They generally accept dialysis treatment but do not choose it $^{35}$. Later on, according to a recent survey, $60 \%$ of dialysis patients sampled reported that they regretted beginning dialysis, and over half of these indicated that they started dialysis because of the urging of their nephrologists ${ }^{33}$.

Inpatient access to palliative care lessens the intensity of care and its cost ${ }^{36,37}$; unfortunately, the vast majority of dialysis patients die in acute care facilities, without ever accessing palliative care services.
Ultimately, the responsibility of caring for ESRD patients and treating their symptoms, either related to the ESRD itself or to comorbid disease, lies with the nephrology team. Nephrology programs and dialysis providers need to develop and integrate a palliative care strategy for ESRD patients ${ }^{38}$. Non-palliative care specialists should be able to attend to all but the most complex of their patients' palliative care needs ${ }^{36}$.

This project is our answer as a group of nephrologists confronted with these patients on a daily basis. Our commitment is to present it to the health authorities, trying to contract a reimbursement fee considered fair and adequate for the services provided, seeking a considerable improvement of the last year of life of our patients, a substantial reduction of the National Health System (NHS) expenses, with concomitant relief of public hospitals from this heavy burden.

Disclosure of potential conflicts of interest: none declared

\section{References}

1. Kurella T, et al. Recognition for conservative care in kidney failure. Am J Kidney Dis 2016;68(5):671 $-673$

2. Grubbs V, Moss A, Cohen L, Fischer M, Germain M, Jassal S et al. A palliative approach to dialysis care: A patient-centered transition to the end of life. Clin J Am Soc Nephrol. 2014;9(12):2203-2209.

3. Internet]. Usrds.org. 2020 [cited 6 July 2020]. Available from: https://www.usrds.org/2012/ pres/ASN2H/Collins_USRDS_ASN_2_hour_V1_final.pdf.

4. Murtagh F, Burns A, Moranne $\mathrm{O}$, et al. Supportive care: Comprehensive conservative care in end-stage kidney disease. Clin J Am Soc Nephrol 2016;11:1909-1914.

5. Kurella T, Covinsky K, Chertow $\mathrm{G}$, et al. Functional status of elderly adults before and after initiation of dialysis. N Eng J Med 2009;361:1539-1547.

6. Arnold R, Zeidel M. Dialysis in frail elders. A role for palliative care. N Eng J Med 2009;361:1597$-1598$.

7. Norma no 017/2011 de 28/9/2011- DGS

8. Biesen W, Luijtgaarden $M$, Brown $E$, et al. Nephrologists' perceptions regarding dialysis withdrawal and palliative care in Europe. Nephrol Dial Transplant 2015:30:1951-1958.

9. Morrison RS. Models of palliative care delivery in the United States. Curr Opin Support Palliat Care. 2013;7(2):201-206.

10. Tamura M, Meier D. Five policies to promote palliative care for patients with ESRD. Clin J Am Soc Nephrol 2013;8:1783-1790.

11. Committee on Economic, Social and Cultural Rights (CESCR). General Comment 14, para. 25 Available from: http:// www.refworld org/pdfid/4538838d0.pdf. [Last accessed on 2017 Nov 04.

12. Sara C, Sara D. Palliative and end-of-life care issues in chronic kidney disease. Curr Opin Support Palliat Care 2015;9(1):14-19.

13. Davidson SN, Jhangri GS, Johnson JA. Longitudinal validation of a modified Edmonton symptom assessment system (ESAS) in haemodialysis patients. Nephrol Dial Transplant 2006;21(11):31893195.

14. Davidson SN, Jhangri GS, Johnson JA. Cross sectional validity of a modified Edmonton symptom assessment system in dialysis patients: a simple assessment of symptom burden. Kidney Int 2006:69(9)1621-1625.

15. Murphy E, Murtagh F, Carey I, Sheerin N. Understanding symptoms in patients with advanced chronic kidney disease managed without dialysis: Use of a short patient-completed assessment tool. Nephron Clinical Practice. 2008;111(1):c74-c80.

16. Moskovitch J, Mount P, Davies M. Changes in symptom burden in dialysis patients assessed using a symptom-reporting questionnaire in clinic. Journal of Palliative Care. 2019;35(1):59-65.

17. Rosansky S, Schell J, Shega J, Scherer J, Jacobs L, Couchoud C et al. Treatment decisions for older adults with advanced chronic kidney disease. BMC Nephrology. 2017;18(1): 2758-2765.

18. Couchoud CG, Beuscart JB, Aldigier JC et al. Development of a risk stratification algorithm to improve patient-centered care and decision making for incident elderly patients with end-stage renal disease. Kidney Int 2015;88:1178-1186.

19. Cohen L, Ruthazer R, Moss A, Germain M. Predicting six-month mortality for patients who are on maintenance hemodialysis. Clin J Am Soc Nephrol 2009;5(1):72-79.

20. Da Silva-Gane M, Wellsted D, Greenshields H, Norton S, Chandna S, Farrington K. Quality of life and survival in patients with advanced kidney failure managed conservatively or by dialysis. Clin J Am Soc Nephrol. 2012;7(12):2002-2009.

21. Couchoud C, Hemmelgarn B, Kotanko P, Germain M, Moranne O, Davison S. Supportive care: time to change our prognostic tools and their use in CKD. Clin J Am Soc Nephrol. 2016;11(10):1892 $-1901$

22. Boddana P, Husbands E, Baig I. End of life care in advanced kidney disease; Renal Unit Gloucestershire Hospitals NHS Foundation Trust, October 2017

23. Schmidt RJ, Moss AH. Dying on dialysis: The case for a dignified withdrawal. Clin J Am Soc Nephrol. 2014 Jan 7;9(1):174-180. 
24. Shared decision making in the appropriate initiation of and withdrawal from dialysis, Clinical practice guideline. second edition, Renal Physicians Association, October 2010.

25. Stjernsward J, Foley KM, Ferris FD. The public health strategy for palliative care. J Pain Symptom Manage 2007;33:486-493.

26. Hjermstad MJ, Stein K. "Palliative Care in Europe." Oxford Textbook of Palliative Nursing, 2015; pp. 1087-1110.

27. Radbruch L, Payne S. White paper on standards and norms for hospice and palliative carein Europe: part 1. Eur J Palliat Care. 2009;16:278-289.

28. Moss A, Holley J, Davison S, Dart R, Germain M, Cohen L et al. Palliative care. Am J Kidney Dis. 2004;43(1):172-185.

29. Alt-Epping B et al. On death and dying - An exploratory and evaluative study of a reflective, interdisciplinary course element in undergraduate anatomy teaching. BMC Medical Education. 2014;14(1):14-15.

30. Brennan F et al. Palliative care as a human right. Oxford Textbook of Palliative Nursing, 2015; pp. $1174-1178$.

31. McAdoo SP, Brown EA, Chesser AM, Farrington K, Salisbury EM. Pan-Thames renal audit group; measuring the quality of end of life management in patients with advanced kidney disease: results from the pan-thames renal audit group. Nephrol Dial Transplant. 2012Apr;27(4):1548-1554.

32. Teno JM, Okun SN, Casey V, Welch LC. Toolkit of Instruments to Measure End of Life Care (TIME) Resource Guide: Achieving quality of care at life's end. Center for Gerontology and Health Care Research, Brown University; Center for Life Care, Planning \& Support; Hospice \& Palliative Care of Cape Cod; Picker Institute.
33. Davison SN. End-of- life care preferences and needs: perceptions of patients with chronic kidney disease. Clin J Am Soc Nephrol. 2010 Feb;5(2):195-204.

34. Seow H, Bainbridge D, Bryant D, Guthrie D, Urowitz S, Zwicker V, et al. The CaregiverVoice survey: A pilot study surveying bereaved caregivers to measure the caregiver and patient experience at end-of-life. J Pall Med. 2016;19(7):712-719.

35. Russ A, Kaufman S. Discernment rather than decision-making among elderly dialysis patients. Semin Dial 2012;25:31-32.

36. Grubbs V. Meeting the palliative care needs of maintenance hemodialysis patients. Clin J Am Soc Nephrol 2018;13:1138-1139.

37. Chettiar A, Rath M, Liu S, et al. Association of inpatient palliative care with health care utilization and post discharge outcomes among Medicare beneficiaries with ESKD. Clin J Am Soc Nephrol 2018;13:1180-1187.

38. Davison S. The ethics of end-of-life care for patients with ESRD. Clin J Am Soc Nephrol 2012;7:2049$-2057$.

\section{Correspondence to:}

Ana Farinha, MD

Nephrocare Setubal

email: alpfarinha@gmail.com 